Agron. Mesoam. 29(2):305-314. Mayo-agosto, 2018

ISSN 2215-3608, doi:10.15517/ma.v29i2.29771

http://www.revistas.ucr.ac.cr/index.php/agromeso

\title{
Áreas potenciales para el cultivo de girasol en condiciones de temporal en Guanajuato, México ${ }^{1}$
}

\section{Potential areas for sunflower cultivation under rainfed conditions in Guanajuato, Mexico}

\author{
Enrique López-Rocha², Ana Isabel Mireles-Arriga², Jesús Hernández-Ruiz², Jorge Eric Ruiz-Nieto², \\ Armando Rucoba-García ${ }^{2}$
}

\begin{abstract}
Resumen
En los últimos cinco años en México se registró un incremento de 189,50 a 13 219,84 hectáreas, en la superficie de cultivo de girasol en temporal, particularmente en el estado de Guanajuato se sembró aproximadamente 450 hectáreas en 2016. Esta forma de cultivo requiere de planeación que contemple variables bioclimáticas para el óptimo desarrollo y rendimiento. El objetivo del presente trabajo fue determinar las áreas con potencial bioclimático para el establecimiento del cultivo de girasol en temporal en el estado de Guanajuato. Durante el ciclo otoño-invierno del 2016 se monitorearon y se estratificaron 45 parcelas de girasol, distribuidas en once municipios, en las que se registró el rendimiento asociado a temperatura máxima y mínima, humedad relativa, radiación solar, precipitación y evapotranspiración. Los datos de parcelas con un rendimiento mayor a 1,89 t/ha se utilizaron para generar polígonos con una saturación de 56 puntos de abundancia, para calcular la distribución potencial mediante el programa Maxent, con 23 variables bioclimáticas como predictores. Las variables con mayor contribución porcentual a la distribución potencial fueron: temperatura promedio del cuatrimestre más seco, altitud, régimen de humedad y perfil edáfico. Las áreas potenciales para el establecimiento del cultivo suman una superficie total de 317028 hectáreas, donde el clima predominante es semicálido subhúmedo, temperatura media anual de $20^{\circ} \mathrm{C}$, precipitación media anual de 600 a 800 $\mathrm{mm}$ y una altitud de 500 a $1500 \mathrm{msnm}$.
\end{abstract}

Palabras clave: Helianthus annиus, factores climáticos, zonas climáticas, rendimiento de cultivos.

\begin{abstract}
In Mexico, in the last five years, there was a 189.50 to 13219.84 hectares increase in sunflower crop surface under rainfed conditions. Particularly, in 2016, in the state of Guanajuato, approximately 450 hectares were planted. Cultivation under rainfed conditions requires planning including bioclimatic variables to reach the optimal crop yield and development. This work aims to identify bioclimatic potential areas for sunflower cultivation under rainfed conditions in Guanajuato. In autumn-winter 2016, 45 sunflower fields (distributed in eleven towns) were monitored
\end{abstract}

1 Recibido: 12 de julio, 2017. Aceptado: 13 de noviembre, 2017. Este trabajo se realizó con las actividades de extensionismo, de los profesores del programa educativo Agronegocios, Campus Irapuato-Salamanca, Universidad de Guanajuato, México.

2 Universidad de Guanajuato, División de Ciencias de la Vida. Km 9 carretera Irapuato-Silao, Ex Hacienda. El Copal, Irapuato, Guanajuato. 36500 México.vrelopezr@hotmail.com, ana.mireles@ugto.mx, hernandez.jesus@ugto.mx (autor para correspondencia), jorge.ruiz@ugto. mx, arucoba@gmail.com 
and stratified. Crop yield associated to maximum and minimum temperature, relative humidity, solar radiation, precipitation and evapotranspiration were recorded. The data from fields with higher yield than $1.89 \mathrm{t} / \mathrm{ha}$ were used to generate polygons with a saturation of 56 abundance points, to calculate the potential distribution using the Maxent software, with 23 bioclimatic variables as predictors. The variables with the highest contribution percentage to the potential distribution were: average temperature of the driest quarter, altitude, moisture regime, and edaphic profile. The potential areas for sunflower cultivation had a surface of 317028 hectares where the predominant climate is sub-humid warm, the annual mean temperature is $20^{\circ} \mathrm{C}$, the annual mean rainfall is 600 to $800 \mathrm{~mm}$, and the altitude ranges from 500 to 1500 masl.

Keywords: Helianthus annuus, climatic factors, climate zones, crop yield.

\section{Introducción}

El girasol (Helianthus annuus L.) fue domesticado en México (Lentz et al., 2008b) y constituye una especie de múltiples propósitos, que puede ser aprovechado en la producción de aceite para la alimentación humana y animal, en forma ornamental y en rotación de cultivos como abono verde, por lo que, es uno de los principales cultivos oleaginosos del mundo (Davey y Jan, 2010).

La producción mundial de girasol en 2016 fue de 45,75 millones de toneladas, donde los principales productores fueron Ucrania (30,6\%), Rusia (24,0\%), Argentina (7,4\%) y China (6,2\%). México aportó solo el $0,28 \%$ de la producción mundial de girasol (USDA, 2017); sin embargo, la superficie para este cultivo ha tenido un aumento considerable de 54 ha en el año 2000 a 13 219,84 ha en el 2015, donde los estados con mayor superficie sembrada son Zacatecas, Tamaulipas, San Luis Potosí, Durango y Guanajuato. De los cuales San Luis Potosí y Guanajuato presentan los rendimientos más elevados a nivel nacional con 1,47 t/ha (SIAP, 2017).

El aumento de superficie sembrada debe contar con la planeación e información básica sobre suelo, clima y demás factores que pueden afectar el rendimiento del cultivo establecido (Suárez, 2014), debido a que, los ciclos vegetativos y los rendimientos de las plantas cultivadas están vinculados con numerosos elementos del entorno, como variables bioclimáticas que forman patrones lógicos de la estructura y función de un ecosistema (Scarrow, 2017). De igual manera, con cultivos establecidos en condiciones de temporal la principal fuente de agua utilizable es la lluvia, el suministro oportuno, cantidad y distribución en diferentes fases de crecimiento es importante para determinar las zonas óptimas para la agricultura en una región (Coras-Merino et al., 2005). Para ello, es necesario desarrollar e instrumentar métodos adecuados para caracterizar el clima y su impacto sobre la producción agrícola (Medina, 1995; Altieri y Nicholls, 2009). El uso de los sistemas de información geográfica representa una herramienta para realizar estudios sobre el análisis espacial de la disponibilidad de tierras agrícolas (Abolina et al., 2015), el cual se puede combinar con análisis de los patrones espaciales de presencia y ausencia que se utilizan comúnmente en los modelos de distribución de especies (Swenson, 2008; Hernández-Ruíz et al., 2016; Hernández et al., 2017).

Bajo la premisa, que la probabilidad de presencia es una interpretación de la medida de similitud ambiental, la cual se interpreta como un valor de idoneidad ambiental para el desarrollo favorable de una especie (Mateo et al., 2011), el objetivo del presente trabajo fue determinar las áreas con potencial bioclimático para el establecimiento del cultivo del girasol, en condiciones de temporal en Guanajuato, México. 


\section{Materiales y métodos}

Durante el ciclo agrícola otoño-invierno 2016, con los datos de la red de estaciones meteorológicas de la Fundación Guanajuato Produce A.C., se monitorearon 45 parcelas de girasol, distribuidas en once municipios del Estado, en las cuales se realizó un barbecho y dos pasos de rastra (cruzados), nivelación del terreno y trazo de surcos de 76 a $80 \mathrm{~cm}$, donde se estableció el híbrido SYN3950 HO (el cual se caracteriza por producir semillas con altas concentraciones de aceite oleico), a una densidad de siembra de 56000 plantas/ha. Se utilizó una dosis de fertilización 80-60-00, a la siembra se aplicó el 50\% de la dosis nitrogenada y el total del fósforo. En la primera escarda se aplicó el 50\% restante del nitrógeno. Se realizó una aplicación foliar de boro al inicio de la floración a razón de 0,5 a $1 \mathrm{~kg}$ en 2001 de agua por hectárea.

En las parcelas se registró el rendimiento $(\mathrm{kg} / \mathrm{ha})$. De la fecha de siembra a la etapa fenológica madurez fisiológica (R9), de acuerdo con Schneiter y Miller (1981), la cual tuvo un lapso en promedio de 150 días, se obtuvieron las variables: temperatura máxima y mínima $\left({ }^{\circ} \mathrm{C}\right)$, humedad relativa máxima y promedio $(\%)$, radiación solar máxima $\left(\mathrm{W} / \mathrm{m}^{2}\right)$, radiación acumulada $\left(\mathrm{Mj} / \mathrm{m}^{2}\right)$, velocidad del viento promedio $(\mathrm{km} / \mathrm{h})$ precipitación acumulada $(\mathrm{mm})$, evapotranspiración acumulada $(\mathrm{mm})$ y rendimiento $(\mathrm{t} / \mathrm{ha})$.

Se consideró el rendimiento de cada parcela en toneladas por hectárea ( $\mathrm{t} / \mathrm{ha})$, para realizar una estratificación por cuantiles (Q1, Q2, Q3), y ubicar las parcelas que presentaron el mayor rendimiento, las cuales se georreferenciaron con base a los cuatro vértices que conforman el polígono de la parcela. Con los datos de los vértices se consideró una saturación de 56 puntos de abundancia (número de presencias registradas para girasol por cada parcela), dado que con valores altos de ocurrencia se pueden generar aproximaciones efectivas del modelo y realizar inferencias de cultivos agrícolas (Heumann et al., 2011). Con los puntos de abundancia se generó una base de datos, y mediante la aplicación del programa Maxent versión del software 3.3.3 (Phillips et al., 2006; Mbatudde et al., 2012), se predijo la distribución potencial para el cultivo de girasol en condiciones de temporal.

Se utilizó un total de veintitrés variables como predictores en modelación de distribución de especies (Cuadro 1), 19 bioclimáticas de resolución espacial de 0,5 minutos de arco se descargaron de la base de datos WorldClim (2016). Los datos digitales de elevación (CEM; $30 \mathrm{~m}$ de resolución) se obtuvieron del Instituto Nacional de Estadística y Geografía (INEGI, 2017). Se utilizaron capas "shapefile" de uso de suelo y vegetación de México (INEGI, 2013), régimen de humedad del suelo (Maples-Vermeersch, 1992) y continuo nacional de datos vectoriales edafológicos (INEGI, 2001), las cuales se convirtieron en formato raster.

\section{Resultados}

La estratificación realizada, agrupó a parcelas de un primer estrato con un rendimiento menor a los $868 \mathrm{~kg} / \mathrm{ha}$, donde la temperatura promedio fue de $26,73{ }^{\circ} \mathrm{C}$, la precipitación acumulada de $423,43 \mathrm{~mm}$ y evapotranspiración acumulada de $525 \mathrm{~mm}$. El segundo estrato agrupó la mayoría de parcelas, en las cuales el rendimiento estuvo en un rango de 869 a $1869 \mathrm{~kg} / \mathrm{ha}$, donde la temperatura promedio fue de $26,64{ }^{\circ} \mathrm{C}$, la precipitación acumulada de 439,04 $\mathrm{mm}$ y evapotranspiración acumulada de $530,80 \mathrm{~mm}$. El tercer estrato se conformó por parcelas con un rendimiento mayor a $1896 \mathrm{~kg} / \mathrm{ha}$, donde la temperatura promedio fue de $26,65^{\circ} \mathrm{C}$, la precipitación acumulada de 433,62 mm, y la evapotranspiración acumulada de $525 \mathrm{~mm}$. Los rendimientos más altos se registraron con una evapotranspiración acumulada de 530,80 mm, radiación máxima 892,58 W/m² y una precipitación acumulada de 433,62 mm.

De las veintitrés variables utilizadas como predictores para el modelo de zonas potenciales, basado en las parcelas con mayor rendimiento ( $>1,89$ t/ha), nueve contribuyeron a determinar la estimación del modelo (Cuadro 2). Las de mayor importancia fueron temperatura promedio del cuatrimestre más seco $(46,2 \%)$, altitud $(14,5 \%)$, régimen de humedad $(13,1 \%)$ y perfil edáfico $(13 \%)$. 
Cuadro 1. Variables ambientales y bioclimáticas utilizadas para determinar la distribución potencial del cultivo de girasol en temporal, durante el ciclo otoño invierno 2016. Guanajuato, México.

Table 1. Environmental and bioclimatic variables used to determine the potential distribution of sunflower cultivation under rainfed conditions, in autumn-winter 2016. Guanajuato, Mexico.

\begin{tabular}{|c|c|c|}
\hline Código & Descripción de variable & Unidades \\
\hline Bio1 & Temperatura promedio anual & ${ }^{\circ} \mathrm{C}$ \\
\hline Bio2 & Variación diurna de la temperatura & ${ }^{\circ} \mathrm{C}$ \\
\hline Bio3 & Isotermalidad & Adimensionales \\
\hline Bio4 & Estacionalidad de la temperatura & $\mathrm{CV}$ \\
\hline Bio5 & Temperatura máxima promedio del periodo más cálido & ${ }^{\circ} \mathrm{C}$ \\
\hline Bio6 & Temperatura mínima promedio del periodo más frío & ${ }^{\circ} \mathrm{C}$ \\
\hline Bio7 & Variación anual de la temperatura & ${ }^{\circ} \mathrm{C}$ \\
\hline Bio8 & Temperatura promedio del cuatrimestre más lluvioso & ${ }^{\circ} \mathrm{C}$ \\
\hline Bio9 & Temperatura promedio del cuatrimestre más seco & ${ }^{\circ} \mathrm{C}$ \\
\hline Bio10 & Temperatura promedio del cuatrimestre más cálido & ${ }^{\circ} \mathrm{C}$ \\
\hline Bio11 & Temperatura promedio del cuatrimestre más frío & ${ }^{\circ} \mathrm{C}$ \\
\hline Bio12 & Precipitación anual & $\mathrm{mm}$ \\
\hline Bio13 & Precipitación del periodo más lluvioso & $\mathrm{mm}$ \\
\hline Bio14 & Precipitación del periodo más seco & $\mathrm{mm}$ \\
\hline Bio15 & Estacionalidad de la precipitación & $\mathrm{CV}$ \\
\hline Bio16 & Precipitación del cuatrimestre más lluvioso & $\mathrm{mm}$ \\
\hline Bio17 & Precipitación del cuatrimestre más seco & $\mathrm{mm}$ \\
\hline Bio18 & Precipitación del cuatrimestre más cálido & $\mathrm{mm}$ \\
\hline Bio19 & Precipitación del cuatrimestre más frío & $\mathrm{mm}$ \\
\hline Bio20 & Altitud & $\mathrm{m}$ \\
\hline Bio21 & Régimen de humedad del suelo & días \\
\hline Bio22 & Cobertura del suelo & 23 tipos \\
\hline Bio 23 & Perfil edáfico & 19 tipos \\
\hline
\end{tabular}

El establecimiento de girasol en condiciones de temporal, presentó un aumento de la probabilidad de presencia con temperaturas promedio del cuatrimestre más seco que superaron los $33^{\circ} \mathrm{C}$, y esta disminuyó con temperaturas por debajo de los $24^{\circ} \mathrm{C}$. Con respecto a la altitud, el rango óptimo para el establecimiento del cultivo varió entre 500 a $1500 \mathrm{msnm}$, con altitudes por encima de este rango, la probabilidad de desarrollo del cultivo es bajo (Figura 1).

El aumento de días de humedad en el suelo, hace que la probabilidad de presencia de la especie sea mayor, lo que sugiere que es factible establecer el cultivo, principalmente de los 180 a 255 días, este rango corresponde al régimen de humedad ústico, el cual fluctúa de 180 a 270 días (Figura 2). En cuanto el perfil edáfico, se observó que los suelos más aptos para establecer los cultivos son Foezem, Vertisol y Litosol.

El modelado de distribución del cultivo de girasol en Guanajuato, a partir de las once parcelas con un rendimiento mayor a1896 kg/ha, ubicó a dieciocho municipios del Estado, como zonas de mayor aptitud potencial para la producción del cultivo (Figura 3), entre las que destacan: Pénjamo con una superficie de 73356 ha, Yuriria (365,38 ha), Valle de Santiago (347,92 ha), Acámbaro (329,00 ha), Irapuato (311,23 ha), Salvatierra (297,60 ha) y Salamanca $(216,42 \mathrm{ha})$, donde el clima es semicálido subhúmedo, temperatura media anual mayor de $18{ }^{\circ} \mathrm{C}$, temperatura del mes más frío menor de $18{ }^{\circ} \mathrm{C}$, temperatura del mes más caliente mayor de $22{ }^{\circ} \mathrm{C}$, la precipitación del mes más seco es menor a $40 \mathrm{~mm}$, el porcentaje de lluvia invernal del 5\% al 10,2\% del total anual y una precipitación media anual de 600 a $800 \mathrm{~mm}$. 
Cuadro 2. Contribuciones porcentuales de las variables bioclimáticas en los modelos de predicción para girasol, durante el ciclo otoño-invierno 2016, en once municipios de Guanajuato, México.

Table 2. Percentage contributions of the bioclimatic variables in the prediction models for sunflower cultivation, in autumn-winter 2016, in eleven towns of Guanajuato, Mexico.

\begin{tabular}{lc}
\hline Variable & Contribución porcentual \\
\hline Temperatura promedio del cuatrimestre más seco (bio 9) & 46,20 \\
Altitud (bio 20) & 14,50 \\
Régimen de humedad del suelo (bio 21) & 13,10 \\
Perfil edáfico (bio 23) & 13,00 \\
Temperatura promedio del cuatrimestre más lluvioso (bio 8) & 4,70 \\
Variación diurna de la temperatura (bio 2) & 3,00 \\
Precipitación del cuatrimestre más lluvioso (bio16) & 2,60 \\
Estacionalidad de la precipitación (bio15) & 1,80 \\
Precipitación del cuatrimestre más frío (bio19) & 0,90 \\
\hline
\end{tabular}
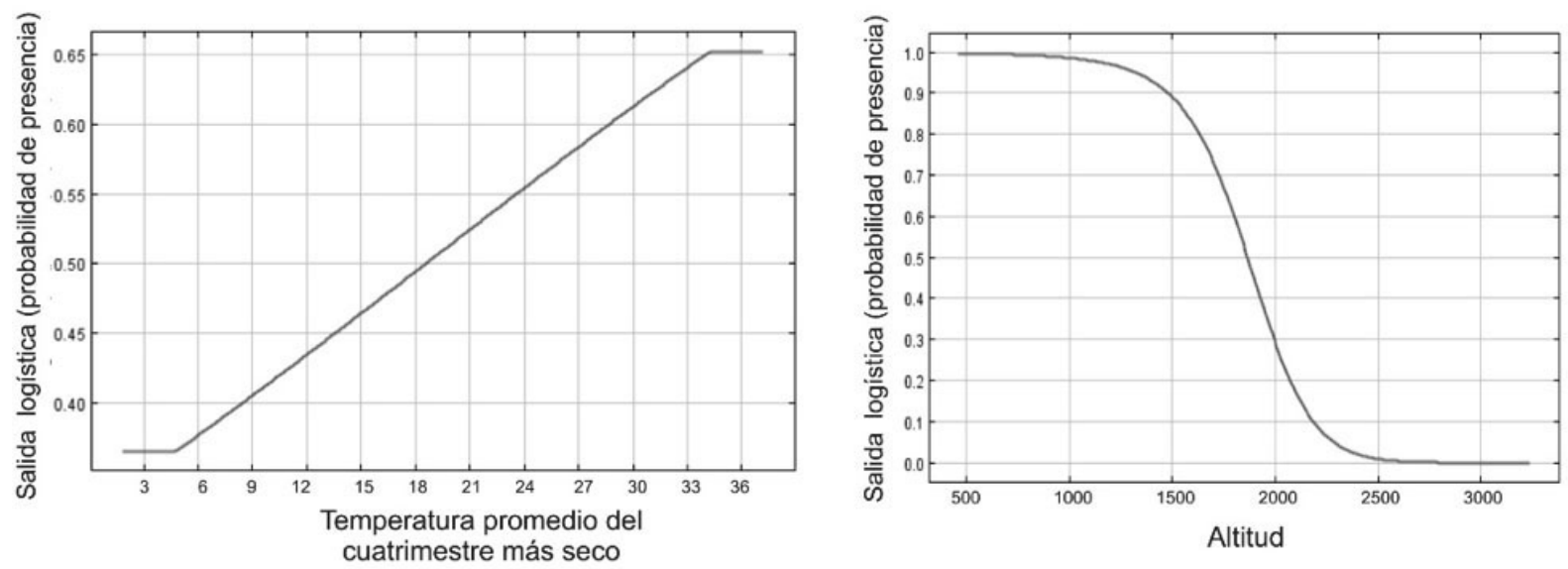

Figura 1. Respuesta del girasol en temporal a las variables temperatura del cuatrimestre más seco y altitud, durante el ciclo otoñoinvierno 2016, en once municipios de Guanajuato, México.

Figure 1. Response of sunflower crop under rainfed conditions to the variables of the driest quarter and altitude, in autumn-winter 2016 , in eleven towns of Guanajuato, Mexico.

Las zonas con alto potencial para el establecimiento de girasol en condiciones temporal ubicadas en Uriangato (6255 ha) y Moroleón (7813 ha), se encuentran en condiciones de clima templado subhúmedo, temperatura media anual entre $12{ }^{\circ} \mathrm{C} \mathrm{y} 18{ }^{\circ} \mathrm{C}$, temperatura del mes más frío entre $-3{ }^{\circ} \mathrm{C}$ y $18{ }^{\circ} \mathrm{C}$.

\section{Discusión}

El rendimiento promedio del cultivo de girasol en México en condiciones de temporal fue de 0,83 t/ha, sin embargo, el estado de Guanajuato registró rendimientos más elevados que variaron de 0,9 t/ha en el municipio de San Francisco del Rincón hasta 2,35 t/ha en los municipios de Pueblo Nuevo y Jaral del Progreso (SIAP, 2017). 

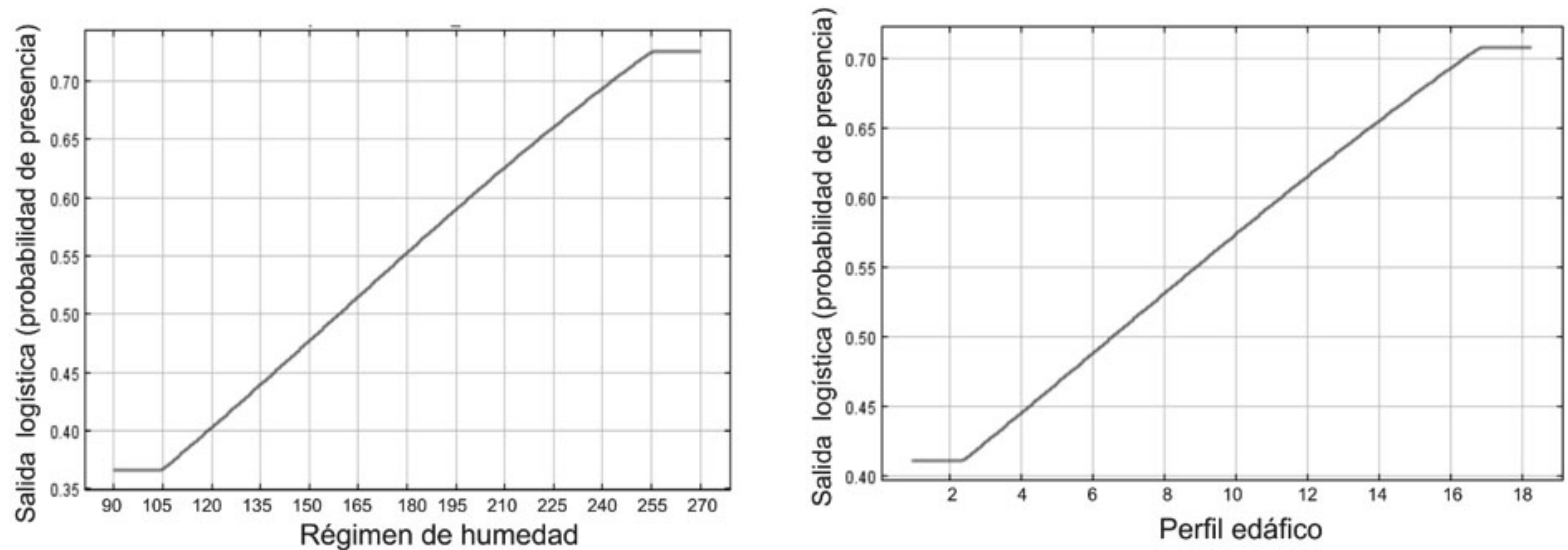

Figura 2. Respuesta del cultivo de girasol a las variables de régimen de humedad y perfil edáfico, durante el ciclo otoño-invierno 2016, en once municipios de Guanajuato, México.

Figure 2. Response of sunflower crop under rainfed conditions to the variables of moisture regime and edaphic profile, in autumnwinter 2016, in eleven towns of Guanajuato, Mexico.

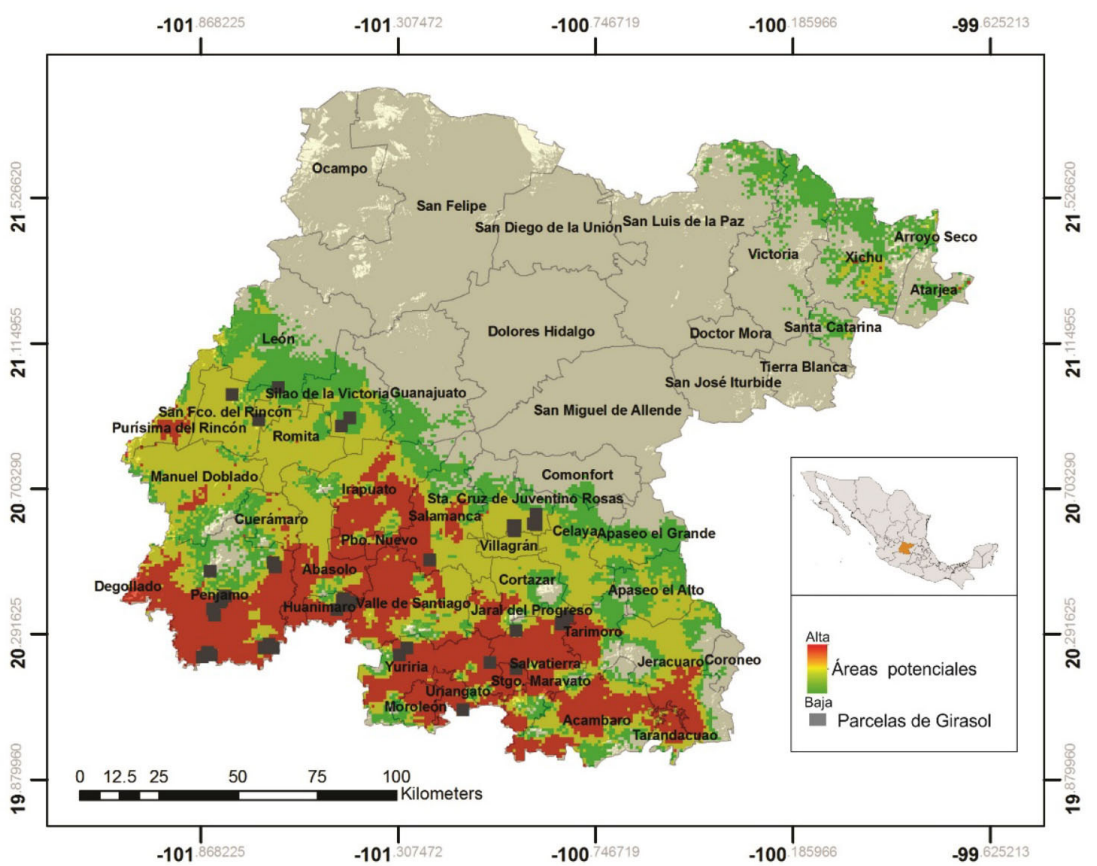

Figura 3. Áreas potenciales para el establecimiento del cultivo de girasol en condiciones de temporal para el estado de Guanajuato, México. 2016.

Figure 3. Potential areas for sunflower cultivation under rainfed conditions in Guanajuato, Mexico. 2016.

Por lo cual, los rendimientos registrados en las parcelas evaluadas se encuentran por encima de la media del rendimiento nacional y estatal, si se considera el estrato de las parcelas que obtuvieron el rendimiento mayor a 1,89 t/ha, las cuales fueron consideradas para estimar la idoneidad ambiental para el desarrollo favorable del cultivo. 
El aumento de la evapotranspiración acumulada, radiación máxima y precipitación acumulada se relacionó con el incremento del rendimiento del cultivo del girasol, esto debido a que, son variables cuantitativas relacionadas directamente con el rendimiento de cultivos, que definen las exigencias de las plantas (Reyna et al., 1997). Aunado que, el girasol se caracteriza por un potencial fotosintético muy elevado, sobre todo en las hojas jóvenes, así como altas tasas de foto-respiración (pérdidas de sustratos carbonados) y de transpiración (pérdida de agua). Lo que favorece a tasas de crecimiento muy elevadas y presenta un nivel de saturación lumínica muy alto (GranadosRamírez et al., 2004).

El programa Maxent se ha utilizado para el modelado de nichos ecológicos de poblaciones silvestres de girasol, donde utilizaron como base diecinueve variables bioclimáticas (Lentz et al., 2008a), las cuales se contemplaron para este estudio y adicionalmente se emplearon las variables: altitud, régimen de humedad de suelo, cobertura del suelo y perfil edáfico, considerando que se estimaron áreas potenciales para el establecimiento de un cultivo, donde la probabilidad de presencia se interpretó como una medida de similitud ambiental y valor de idoneidad ambiental para el desarrollo favorable de la especie (Mateo et al., 2011).

Se reportó que el girasol silvestre tiene una tolerancia para diferentes tipos de suelo y una gama amplia de pH de 5,7 a 8 (Lentz et al., 2008a), sin embargo, en condiciones de cultivo, las plantas demandan características específicas para su óptimo desarrollo y rendimiento agrícola. En México se recomienda para su establecimiento los tipos de suelo Litosoles y Vertisoles (Hernández y Paredes, 2004). Este resultado concuerda con la estimación de la probabilidad de presencia realizada, dado que las zonas potenciales identificadas para el cultivo de girasol en Guanajuato, poseen suelos Foezem, Vertisol y Litosol.

De manera natural, el girasol se distribuye en una amplia variedad de condiciones climáticas, desde el sur de Canadá hasta la meseta central de México (Lindstrom et al., 2006). El establecimiento en condiciones de cultivo requiere de clima tropical, subtropical o templado, con precipitaciones de 400 a $550 \mathrm{~mm}$ anuales, temperatura anual promedio $23{ }^{\circ} \mathrm{C}$ y un rango de altitud de 0 a $1900 \mathrm{msnm}$ (García et al., 1996). Estos resultados son contrastantes debido a que, la probabilidad de la presencia óptima que se estimó en este estudio fue en condiciones de clima semicálido subhúmedo, con precipitación media anual de 600 a $800 \mathrm{~mm}$ anuales y una elevación de 500 a 1500 msnm. Estas condiciones se encuentran principalmente al sureste y suroeste del Estado, en esta zona los municipios de Silao, Cuerámaro y Abasolo, fueron reportados por Hernández y Paredes (2004) como zonas idóneas para el establecimiento del cultivo, aunque se determinó por un método cualitativo de sobreposición de capas que consideraron las variables clima, suelo y topografía.

El estudio realizado por Granados-Ramírez et al. (2004) determinó que las zonas ubicadas en la porción noroeste y central del Estado de Guanajuato, son aptas para establecer girasol en condiciones de temporal, y que el relieve, suelos poco desarrollados y condiciones termopluviométricas en la porción sureste, no son propicias para el desarrollo del cultivo. Sin embargo, este resultado pudo estar influenciado por el método de categorización cualitativa de sobreposición de un número limitado de variables, las cuales fueron temperatura media, máxima, mínima, precipitación media y porcentaje de sequía relativa. A diferencia del presente estudio, en el cual se consideró diecinueve variables bioclimáticas, datos de elevación, régimen de humedad, perfil edáfico y cobertura del suelo, en los cuales la probabilidad de presencia fue determinada por el programa Maxent, como valor de idoneidad ambiental para el desarrollo favorable del cultivo (Phillips et al., 2009).

Los modelos predictivos de distribución de especies son importantes para una variedad de aplicaciones en ecología y conservación (Mateo et al., 2011), por ejemplo, se utilizan en conservación de especies silvestres como V. planifolia, con el objetivo de generar estrategias de preservación del acervo genético (Hernández-Ruiz et al., 2016); se aplican al estudio de propagación de especies invasoras, como la dispersión de plantas de las familias Asteraceae, Amaranthaceae y Phytolaccaceae (Horvitz et al., 2017); coadyuvan a conocer los impactos del cambio climático (Plutzar et al., 2016) y los patrones espaciales de la diversidad de especies (López-Martínez et al., 2016). Sin embargo, son pocas las veces que se ha utilizado para tratar de determinar la idoneidad ambiental 
para el establecimiento de cultivos como el de papaya en México (Hernández-Ruiz et al., 2017), dado que para la obtención de los valores de ocurrencia se deben emplear una alta cantidad de puntos de abundancia para crear una aproximación efectiva del modelo. Esto dado que, los modelos como Maxent (solo de presencia) tienen un gran potencial para el modelado de idoneidad de cultivo donde no existen verdaderas ausencias, porque un determinado cultivo podría establecerse en la mayoría o en todas las ubicaciones de un área de estudio (Heumann et al., 2011).

\section{Conclusiones}

Los rendimientos registrados durante la evaluación de las parcelas cultivadas de girasol superó la media de producción nacional de México, por lo que, se consideró que existe una aptitud bioclimática en el Estado de Guanajuato para el establecimiento del cultivo de girasol.

El modelado de distribución potencial realizado con el programa Maxent, mediante la interpretación de la probabilidad de presencia, como un valor de idoneidad ambiental para el desarrollo del cultivo de girasol, permitió identificar áreas potenciales para el establecimiento del cultivo en condiciones de temporal las cuales suman una superficie total de 317028 ha.

Se infiere que el modelado de distribución potencial de especies y la idoneidad del hábitat, puede servir como una herramienta para la toma de decisiones del posible establecimiento de cultivos, ya que los ciclos vegetativos y los rendimientos de las plantas cultivadas están vinculados a variables ambientales del entorno, como la temperatura, precipitación, altitud y radiación solar.

\section{Literatura citada}

Abolina, E., T.A. Volk, and D. Lazdina. 2015. GIS based agricultural land availability assessment for the establishment of short rotation woody crops in Latvia. Biomass Bioenerg. 72:263-272. doi:10.1016/j.biombioe.2014.10.026

Altieri, M.A., y C.I. Nicholls. 2009. Cambio climático y agricultura campesina: impactos y respuestas adaptativas. Leisa Rev. Agroecol. 24:13-22.

Coras-Merino, P.M., R. Arteaga-Ramírez, R, y M. Vázquez-Peña. 2005. Análisis de frecuencias de lluvias máximas con fines de drenaje superficial local, Tabasco, México. Terra Latinoam. 23:113-120.

Davey, M.R., and M. Jan. 2010. Sunflower (Helianthus annuus L.): genetic improvement using conventional and in vitro technologies. J. Crop Improv. 24:349-391. doi:10.1080/15427528.2010.500874

García, N.H., A.C. Mejía, R.D. García, R.M. Chacón, y A.R. Gonzales. 1996. Zonas potenciales para la producción de las principales especies vegetales en el estado de Guanajuato. INIFAP, Celaya, Gto., MEX.

Granados-Ramírez, R., T. Reyna-Trujillo, J. Soria-Ruiz, y Y. Fernández-Ordóñez. 2004. Aptitud agroclimática en la mesa central de Guanajuato, México. Invest. Geográf. 54:24-35.

Hernández, M.M., y M.R. Paredes. 2004. Guía para la producción de girasol. En: M.A. Mandujano et al., editores, Guía para la producción de cultivos alternativos en Guanajuato. Libro Técnico No 5. INIFAP, Celaya, Gto. MEX. p. 138-156.

Hernández-Ruiz, J., E. Espinosa-Trujillo, F. Palemon-Alberto, y R. Guzmán-Mendoza. 2017. Distribución potencial de cultivares de Carica papaya L. en México. En: I. Covarrubias-Gutiérrez et al., editores, Memoria del V Congreso Internacional y XIX Congreso Nacional de Ciencias Agronómicas. Editorial Universidad de Chapingo. Estado de México, MEX.p. 456-458. 
Hernández-Ruiz, J., B.E. Herrera-Cabrera, A. Delgado-Alvarado, V.M. Salazar-Rojas, A. Bustamante-González, J.E. CamposContreras, and J. Ramírez-Juárez. 2016. Potential distribution and geographic characteristics of wild populations of Vanilla planifolia (Orchidaceae) Oaxaca, Mexico. Rev. Biol. Trop. 64:235-246. doi:10.15517/rbt.v64i1.17854

Heumann, B.W., S.J. Walsh, and P.M. McDaniel. 2011. Assessing the application of a geographic presence-only model for land suitability mapping. Ecol. Inform. 6:257-269. doi:10.1016/j.ecoinf.2011.04.004

Horvitz, N., R. Wang, F.H. Wan, and R. Nathan. 2017. Pervasive human-mediated large-scale invasion: analysis of spread patterns and their underlying mechanisms in 17 of China's worst invasive plants. J. Ecol.105:85-94. doi:10.1111/13652745.12692

INEGI (Instituto Nacional de Estadística y Geografía). 2001. Conjunto de datos vectoriales edafológico, escala 1:250000 Serie II. (Continuo Nacional)', escala: 1:250000. INEGI, MEX. http://www.conabio.gob.mx/informacion/gis/ (consultado 2 feb. 2017).

INEGI (Instituto Nacional de Estadística y Geografía). 2013. Conjunto de datos vectoriales de uso de suelo y vegetación, escala 1:250 000, serie V (capa unión)', escala: 1:250000. $2^{\text {da }}$ ed. Aguascalientes, México. INEGI, MEX. http://www.conabio. gob.mx/informacion/gis/ (consultado 2 feb. 2017).

INEGI (Instituto Nacional de Estadística y Geografía). 2017. Datos de relieve: continuo de elevaciones mexicano 3.0 (CEM; $30 \mathrm{~m}$ de resolución). INEGI, MEX. http://www.inegi.org.mx/geo/contenidos/datosrelieve/continental/descarga.aspx (consultado 2 feb. 2017).

Lentz, D.L., R. Bye, and V. Sánchez-Cordero. 2008a. Ecological niche modeling and distribution of wild sunflower (Helianthus annuus L.) in Mexico. Int. J. Plant. Sci. 169:541-549. doi:10.1086/528754

Lentz, D.L., M.D. Pohl, J.L. Alvarado, S. Tarighat, and R. Bye. 2008b. Sunflower (Helianthus annuus L.) as a pre-Columbian domesticate in Mexico. PNAS 105:6232-6237. doi:10.1073/pnas.0711760105

Lindstrom, L.I., C.N. Pellegrini, L.A.N. Aguirrezábal, and L.F. Hernández. 2006. Growth and development of sunflower fruits under shade during pre-and early post-anthesis period. Field. Crops Res. 96:151-159. doi:10.1016/j.fcr.2005.06.006

López-Martínez, V., G. Sánchez-Martínez, D. Jiménez-García, N.B. Pérez-De-la-O, and T.W. Coleman. 2016. Environmental suitability for Agrilus auroguttatus (Coleoptera: Buprestidae) in Mexico using MaxEnt and database records of four Quercus (Fagaceae) species. Agric. For. Entomol. 18:409-418. doi:10.1111/afe.12174

Maples-Vermeersch, M. 1992. Regímenes de humedad del suelo en Hidrogeografía IV.6.2 Atlas Nacional de México. Vol. II. Escala 1:4000000. Instituto de Geografía, y UNAM, MEX.

Mateo, R.G., A.M. Felicísimo, y J. Muñoz. 2011. Modelos de distribución de especies: Una revisión sintética. Rev. Chil. Hist. Nat. 84:217-240. doi: 10.4067/S0716-078X2011000200008

Mbatudde, M., M. Mwanjololo, E.K. Kakudidi, and H. Dalitz. 2012. Modelling the potential distribution of endangered Prunus africana (Hook. f.) Kalkm. in East Africa. Afr. J Ecol. 50:393-403. doi:10.1111/j.1365-2028.2012.01327.x

Medina, B.M.P. 1995. Metodología para obtener índices agroclimáticos. Tesis Lic., Colegio de Geografía, UNAM, MEX.

Phillips, S.J., M. Dudík, J. Elith, C.H. Graham, A. Lehmann, J. Leathwick, and S. Ferrier. 2009. Sample selection bias and presence-only distribution models: implications for background and pseudo-absence data. Ecol. Appl. 19:181-197. doi:10.1890/07-2153.1

Phillips, S.J., M. Dudík, and R.E. Schapire. 2006. Maxent software for species habitat modeling. Modeling Version 3.3.1. Princeton University, NJ, USA.

Plutzar, C., K.H. Erb, V. Gaube, H. Haberl, and F. Krausmann. 2016. Of birds and bees: Biodiversity and the colonization of ecosystems. In: H. Haberl et al., editors, Social ecology. Springer International Publishing, SUI. p. 375-388. 
Reyna, T., J.M. Fernández, T. López, y A.L. Hernández. 1997. Zonificación pluviométrica para el cultivo del Amaranthus spp. en la provincia de Pinal del Río e Isla de la Juventud, Cuba. Invest. Geográf. 35:135-154.

Scarrow, R. 2017. Climate change: Variance in crop yields. Nature Plants 3, 17059. doi:10.1038/nplants.2017.59

Schneiter, A.A., and J.F. Miller. 1981. Description of sunflower growth stages. Crop Sci. 21:901-903.

SIAP (Servicio de Información Agroalimentaria y Pesquera). 2017. Anuario estadístico de la producción agrícola. SIAP, MEX. http://infosiap.siap.gob.mx/aagricola_siap_gb/icultivo/index.jsp (consultado 1 jun. 2017).

Suárez, G.M. 2014. Apuntes sobre la zonificación agroecológica de los cultivos. Particularidades en Cuba. Cultivos Trop. 35(4):36-44.

Swenson, N.G. 2008. The past and future influence of geographic information systems on hybrid zone, phylogeographic and speciation research. J. Evol. Biol. 21:421-434. doi:10.1111/j.1420-9101.2007.01487.x

USDA. 2017. Production, supply \& distribution, electronic database. https://www.fas.usda.gov/databases/production-supplyand-distribution-online-psd (accessed 4 Jun. 2017).

WorldClim. 2016. Electronic database. http://www.worldclim.org. (accessed 2 feb. 2017). 